\title{
BMJ Open Internet- and mobile-based aftercare and follow-up for mental disorders: protocol of a systematic review and meta-analysis
}

\author{
Severin Hennemann, ${ }^{1}$ Sylvia Farnsteiner, ${ }^{1}$ Lasse Sander ${ }^{2}$
}

To cite: Hennemann S, Farnsteiner S, Sander L. Internet- and mobile-based aftercare and follow-up for mental disorders: protocol of a systematic review and meta-analysis. BMJ Open 2017;7:e016696. doi:10.1136/ bmjopen-2017-016696

- Prepublication history and additional material are available. To view these files please visit the journal online (http://dx.doi. org/10.1136/bmjopen-2017016696).

Received 3 March 2017 Revised 31 May 2017

Accepted 1 June 2017

CrossMark

${ }^{1}$ Department of Clinical

Psychology, Psychotherapy and Experimental Psychopathology, University of Mainz, Germany ${ }^{2}$ Department of Rehabilitation Psychology and Psychotherapy, Institute of Psychology, University of Freiburg, Germany

Correspondence to Dr Severin Hennemann; s. hennemann@uni-mainz.de

\section{ABSTRACT}

Introduction Mental disorders are characterised by a high likelihood of symptom recurrence or chronicity. Thus, in the vulnerable post-discharge phase, aftercare and follow-up aim at stabilising treatment effects, promoting functionality and preventing relapse or readmission. Internet- and mobile-based interventions may represent low threshold and effective extensions to aftercare in tertiary prevention of mental disorders.

Objectives The planned systematic review and metaanalysis aims to synthesise and analyse existing evidence on the effectiveness of psychological internet- and mobile-based aftercare or follow-up in maintaining treatment effects and/or preventing recurrence in adults with mental disorders. Methods and analysis Electronic databases (Psyclnfo, MEDLINE and Cochrane Central Register of Controlled trials) will be searched systematically, complemented by a hand-search of ongoing trials and reference lists of selected studies. Data extraction and evaluation will be conducted by two independent researchersand quality will be assessed with the Cochrane risk of bias tool. Eligibility criteria for selecting studies will be: randomised controlled trials of internet-based and mobile-based, psychological aftercare and follow-up for the tertiary prevention of mental disorders in an adult population. Primary outcome will be symptom severity. Secondary outcomes will be symptom or disorder recurrence rate, rehospitalisation rate, functionality, quality of life or adherence to primary treatment. Further data items to be extracted will be: study design, intervention and technical characteristics, type of mental disorder or clinical symptom to be treated, target population items, setting, treatment engagement and assessment of additional outcome variables. Meta-analytic pooling will be conducted when data of included studies are comparable in terms of study design, intervention type, endpoints, assessments and target mental disorder. Cumulative evidence will be evaluated according to the Grading of Recommendations Assessment, Development and Evaluation framework.

Ethics and dissemination Ethics approval is not required. Results from this review will be published in peer-reviewed journals and presented at international conferences. Trial registration number PROSPERO CRD42017055289.

\section{INTRODUCTION}

Mental disorders are not only highly prevalent ${ }^{1}$ but are also characterised by frequent

\section{Strengths and limitations of this study}

- This review performs a sensitive search in electronic databases on digital technologies in tertiary prevention and will be the first to evaluate the effectiveness of internet- and mobile-based aftercare and follow-up in maintaining treatment effects or preventing recurrence in adults with mental disorders.

- Heterogeneity of studies in terms of clinical, methodological or statistical aspects will be considered carefully.

- The differentiated findings will provide clinicians and public health policy makers with a valuable overview of the feasibility of internet- and mobilebased interventions in tertiary prevention of mental disorders.

- The present protocol follows the preferred reporting items for systematic review and meta-analysis protocols guidelines.

- We plan to assess the confidence in the cumulative evidence with the Grading of Recommendations Assessment, Development and Evaluation system.

recurrence during lifetime or chronic courses. $^{2-5}$ Adverse effects of recurrence or chronicity can be severe and include elevated readmission rates, ${ }^{6}$ early retirement, ${ }^{7}$ reduced quality of life ${ }^{8}$ and increased mortality. ${ }^{9}$

Within all areas of healthcare, tertiary prevention is paramount to monitor and manage symptoms, prevent relapse and promote health and functioning in persons with mental disorders. ${ }^{10}$ In terms of continuous care, tertiary prevention may therefore comprise psychosocial, pharmacological or vocational rehabilitation, aftercare, follow-up or maintenance treatment. In particular, the transition after inpatient treatment can be considered a vulnerable phase, ${ }^{11}$ in which convalescents have to transfer and maintain health behaviour, initiate change and are confronted with various individual, social or occupational challenges. $^{12}$ 
Meta-analytic evidence suggests the efficacy of cognitive behavioural therapy (CBT) ${ }^{13}{ }^{14}$ psychosocial interventions, ${ }^{15} 16$ pharmacological maintenance treatment ${ }^{17}$ or psychosomatic rehabilitation ${ }^{18}$ in reducing symptom severity, relapse rates and promoting functionality or medication adherence ${ }^{1920}$ in mental disorders following acute treatment.

However, implementation strategies of aftercare are very heterogeneous and vary between different healthcare systems, mental disorders and treatment modalities. In this regard, studies in patients with psychiatric disorders or chronic pain indicate an insufficient prescription of aftercare by clinicians. ${ }^{21}{ }^{22}$ Other studies suggest a limited uptake or adherence of psychosocial or medical maintenance treatment in convalescents. ${ }^{23-26}$ Reasons for non-participation in psychosocial aftercare may include long waiting times, ${ }^{27}$ pessimistic treatment expectancies ${ }^{24}$ or various organisational barriers. ${ }^{22}$ On the other hand, insufficient resources of healthcare systems and medical costs may further limit an extensive implementation and lead to gaps in continuity of care. ${ }^{28}$

In an effort to overcome these limitations, internet-delivered health promotion and treatment options for mental disorders have been developed particularly in the last decade. Internet- and mobile-based interventions (IMIs) can be defined as "health-related services and systems, carried out over a distance by means of information and communications technologies, for the purpose of global health promotion, disease control and healthcare' (p1). ${ }^{29}$ IMIs can be categorised by technical implementation (eg, personal computer, smartphone and wearables), content (eg, education, monitoring and behaviour change), localisation in the healthcare process (eg, prevention, stand-alone interventions, blended- or aftercare), amount of human support (self-administered/automatised, selfhelp with minimal guidance and online therapy) or therapeutic contact (eg, email, short messaging service and live chat/video) ${ }^{30}$ IMIs can be administered cost-effectively and without local or temporal boundaries. ${ }^{31} 32$ As internet access and use are growing constantly across countries and age groups, ${ }^{33}$ IMIs also represent widely accessible instruments.

A growing amount of evidence suggests efficacy of web-based psychotherapeutic interventions for a wide range of mental conditions. ${ }^{34} 35$ One of the first transdiagnostic reviews by Barak and colleagues ${ }^{34}$ found small to large effect sizes of IMIs ranging from standardised mean difference $(\mathrm{SMD})=0.32$ (depression, $\mathrm{n}=16$ ) to $\mathrm{SMD}=0.88$ (post-traumatic stress disorder (PTSD), n=3). Further reviews focused on IMIs as stand-alone interventions, including meta-analytic evidence of efficacy in depression $(\mathrm{SMD}=0.56, \mathrm{n}=19),{ }^{36}$ anxiety disorders $(\mathrm{SMD}=1.06$, $\mathrm{n}=28)^{37}$ or PTSD (CBT-based interventions, $\mathrm{SMD}=0.95$, $\mathrm{n}=8) .{ }^{38}$ However, IMIs in psychiatric disorders are less studied, although first randomised controlled trials (RCTs) show promising results. ${ }^{39}$ With regard to the implementation of IMIs in different contexts of healthcare, a recent review by Sander and colleagues ${ }^{40}$ found small to medium cross-diagnostic effect sizes $(\mathrm{d}=0.11-0.76)$ of IMIs in the primary prevention of mental disorders. Furthermore, a review by Niuwenhuijsen $e t a l^{41}$ suggests efficacy of remote interventions (internet- or telephone-based) on return-towork of patients with depression.

Previous studies on internet- or mobile-based aftercare focused on guided, web-based self-help including psychoeducation as well as modular, interactive treatment elements and a certain amount of asynchronous therapist contact. ${ }^{42}{ }^{43}$ Other approaches comprise mobile-based ${ }^{44}$ or synchronous, chat- or video-based aftercare. ${ }^{45}{ }^{46}$ First evidence suggests the efficacy of IMIs in relapse prevention or reduction of symptom severity. ${ }^{42} 46$

However, to the best of our knowledge, no previous systematic review has investigated comprehensive evidence on IMIs as aftercare instruments for adults with mental disorders. Thus, the results of this review will give an overview of this field of research and identify potentials of IMIs for public health policy makers and healthcare providers. The present protocol describes the rationale and design of the systematic review and planned meta-analysis according to the 'preferred reporting items for systematic review and meta-analysis protocols (PRISMA-P) ${ }^{47}$

\section{Objectives}

The aim of this systematic review and meta-analysis is to give a comprehensive overview of RCTs investigating the effectiveness of internet- and mobile-based psychological aftercare (eg, rehabilitation, follow-up and maintenance treatment) in maintaining treatment effects or in preventing symptom or disorder recurrence of mental disorders in adults.

\section{METHODS \\ Eligibility criteria \\ Population}

Studies will be included if they (a) focus on an adult population ( $\geq 18$ years) who (b) have received treatment for a mental disorder or a somatic condition with comorbid mental symptoms within the previous 6 months. Preceding treatment of mental disorders may consist of inpatient or outpatient psychotherapy, psychiatric treatment or medical treatment, delivered by physicians or psychotherapists. Mental disorders must (c) be assessed by a standardised or validated instrument, including standardised interviews (eg, Structured Clinical Interview (SCID) and Composite International Diagnostic Interview (CIDI) ), validated self-reports (eg, Beck Depression Inventory (BDI), Beck Anxiety Inventory (BAI) and Eating Disorder Inventory (EDI)), clinician-rated scales (eg, Hamilton Depression Scale (HAMD) and Global Assessment of Functioning (GAF)) or diagnosis by healthcare professionals.

\section{Study design and interventions}

(d) Only RCTs that are available in full text will be considered. Manuscripts must be published in English or German. Treatment groups should receive a psychological aftercare or follow-up intervention. Following the 
definition by Kampling et $a l^{48}$ psychological interventions (e) may include elements of evidence-based therapy forms (eg, CBT, psychodynamic therapies, behaviour therapy or behaviour modification, systemic therapies, third-wave cognitive behavioural therapies, humanistic therapies or integrative therapies). Interventions may contain symptom monitoring, promotion of adherence to primary treatment (eg, medication compliance), psychoeducation, reinforcement/feedback mechanisms as well as interactive elements or comprise guided/ unguided self-help or comprehensive psychotherapeutic programmes. If symptom monitoring or reminders to treatment adherence are the predominant intervention modality, studies will only be included, if accompanied by a distinguishable psychological intervention element. Treatments not clearly described will be excluded.

(f) Aftercare and follow-up will be defined as interventions following acute treatment designed to monitor or stabilise mental symptoms, identify or manage warning signs of symptom/disorder recurrence or enhance coping strategies to prevent recurrence, relapse or readmission, ${ }^{49}$ support transition and adoption of acquired health behaviour and to promote or preserve health status, thereby reducing the impact of the illness on functioning or quality of life.

(g) Interventions have to be delivered predominantly in an online setting, via internet (web/online) or mobile applications. Interventions may vary in the amount of human support, ranging from unguided self-help, over asynchronous minimal guidance to synchronous therapist contact. $^{50}$

Studies must (h) report a minimum follow-up assessment of the main outcome of 3 months . Follow-up periods of 3-6 months will be categorised as 'short', 6-12 months as 'medium' and above as 'long-term'.

\section{Comparators}

(i) Control groups may receive either no intervention or comprise a waiting list (inactive control group) or include treatment as usual, another form of treatment (eg, faceto-face psychotherapy, phone-delivered treatment, pharmacological/placebo treatment and other forms of psychological interventions) as the active control group.

\section{Exclusion criteria}

Studies will be excluded, if they focus on the prevention of the first onset of a mental disorder or if no distinguishable treatment preceded the intervention under study (stand-alone interventions). Substance-related and addictive disorders will not be included, as this represents another specific research area ${ }^{51} 52$ and treatment rationales are predominantly socioeducational or follow a health behaviour change model rather than psychotherapeutic intervention models.

\section{Information sources and search strategy}

Electronic databases that will be included are MEDLINE, PsycInfo and the Cochrane Central Register of Controlled trials (CENTRAL). A sensitive search strategy will be applied (see online supplementary file 1). The WHO International Clinical Trials Registry Platform will be hand searched to identify ongoing trials. To assure literature saturation, reference lists of included studies will be perused. In case of unclear eligibility or indication of missing or unpublished data, we will contact the principal investigators (PIs) of studies for clarification. Also, when study protocols without a succeeding publication of results are identified, we attempt to contact PI to obtain unpublished results and determine eligibility for inclusion.

\section{Study records}

In a first step, two independent reviewers (SF and $\mathrm{SH}$ ) will screen titles and abstracts of the database search to identify qualified studies. Records will be managed in CITAVI. In a second step, these reviewers will examine full texts in terms of the eligibility criteria. Likewise, the reference lists will be screened against eligibility criteria. In case of disagreement on eligibility, a third reviewer (LS) will be consulted. Inter-rater reliability will be examined to evaluate the consistency of study selection. To illustrate the search and selection process, a flowchart according to the PRISMA- $\mathrm{P}^{47}$ will be provided. Criteria for the exclusion of studies will be reported.

Extracted data of eligible studies will be verified by a second reviewer to assure accuracy. Disagreement will be solved by discussion or by consulting a third reviewer in case of unresolved disagreements. Data extraction forms will be developed and piloted. In case of overlapping or multiple reports, we plan to compare studies with regard to list of authors, sample sizes, treatments or outcomes. In case of unclear or missing information, we will contact PIs with a request to provide these data.

\section{Data items}

The following data items will be extracted for each study: (a) study identification items (first author and year of publication), (b) study design characteristics (eg, sample size, control group, pretreatment, lengths of follow-up assessment and study dropout), (c) intervention characteristics (eg, psychological/therapeutic methods, amount of human guidance, synchronicity of contact and duration of intervention), (d) technical characteristics (eg, internet-based/ mobile-based, devices used and technical prerequisites), (e) type of mental disorder or clinical symptom to be treated, (f) target population items (eg, age and gender), (g) setting (eg, recruitment strategy, nationality, environment and language), (h) treatment engagement (eg, treatment dropout rate, treatment fidelity and adoption of outpatient therapy), (i) assessment of additional outcome variables and (j) clinical outcome (symptom severity, recurrence/incidence rate, rehospitalisation, functionality/ quality of life and adherence to primary treatment).

\section{Outcomes and prioritisation}

Primary outcome will be symptom severity assessed via validated instruments (standardised interviews, self-rated 
or clinician-rated scales) or clinical diagnosis as an indicator of maintenance of treatment effects.

Secondary outcomes will be defined as (a) symptom recurrence or incidence rate of mental disorder under study from post-treatment to latest available follow-up, (b) rehospitalisation rate, (c) indicators of functionality or quality of life and (d) adherence to primary treatment (eg, medication compliance).

In the likely case of multiple assessment instruments for primary or secondary outcome, we will prioritise data as follows: (1) Data from structured interviews will be prioritised. (2) Clinician-rated scales will be preferred over self-report instruments. (3) Self-report questionnaires will be prioritised over diagnosis by health professionals.

When several assessment instruments are used within one study that can be assigned to the same hierarchy level, we will (1) extract outcome of the most frequently used instrument according to eligible studies or (2) if not evident, select randomly. To control for an investigator bias, a second reviewer $(\mathrm{SH})$ will cross-check the extraction process.

\section{Risk of bias in individual studies}

The quality of evidence of each study will be evaluated following the Cochrane risk of bias tool. ${ }^{53}$ The domains to be analysed will be: (a) random sequence generation, (b) allocation concealment, (c) blinding of participants and personnel, (d) blinding of outcome assessment, (e) incomplete outcome data, (f) selective outcome reporting and $(\mathrm{g})$ other threats to validity (eg, treatment fidelity, parallelism of measurement, variance homogeneity at baseline and cointerventions).

As a distinctive feature of psychological interventions, blinding of healthcare providers (in guided IMIs) or patients regarding treatment is not warranted, resulting in a high risk of bias rating of criterion (c). However, outcome assessors can remain unaware of participant's treatment allocation (criterion (d)).

\section{Data synthesis}

\section{Qualitative synthesis}

A narrative synthesis will be reported on all included studies and relevant characteristics listed under 'data items' will be qualitatively described. A detailed description of their results on relevant domains will be provided in text and 'summary of findings' tables (comparison against control groups) following PRISMA-P. ${ }^{47}$

\section{Meta-analysis}

The expected heterogeneity of studies in terms of clinical (eg, mental disorder), intervention-related (eg, objective and type of IMI), methodological (eg, comparators and assessment methods) or statistical (eg, comparability of outcome measures) aspects will be considered carefully. Thus, meta-analytic pooling will only be conducted, if comparability of included studies is met in at least three studies. The Cochrane Collaborations' Review Manager will be used. By separating analyses in terms of mental disorders or intervention type, we plan to reduce heterogeneity of pooled estimates. A random-effect model will be used. Only studies with less than substantial statistical heterogeneity by will be pooled. If possible, heterogeneity of study results will be analysed through forest plots and calculating $\mathrm{I}^{2}$ statistics. The degree of heterogeneity will then be categorised according to the guidelines of the risk of bias tool. ${ }^{53}$

For continuous data, we will calculate SMD and 95\% CI. For dichotomous data, we will transform findings into risk ratios. We aim to calculate the number needed to treat (NNT) to further illustrate clinical relevance of the interventions.

Outcome variables (eg, symptom severity scores) will be pooled and further differentiated in terms of 'short', 'medium' or 'long-term' effectiveness when follow-up assessment is reported. Subject to sufficient group size and comparability of assessments, we plan to analyse study-level covariates (eg, type of mental disorder, type of IMI or amount of guidance).

\section{Meta-biases: confidence in cumulative evidence}

We will retrieve study protocols or trial registrations to identify reporting biases. Thereby, we will evaluate whether selective reporting of outcomes is present. A possible small sample bias will be assessed by using a random-effect model. Provided the number of studies is sufficient, we plan to examine a possible publication bias of significant-only studies in funnel plots. We will also search for unpublished or non-significant studies.

We plan to rate the cumulative evidence according to the Grading of Recommendations Assessment, Development and Evaluation (GRADE) ${ }^{54}$ in terms of study limitations, inconsistency of results, indirectness of evidence and imprecision of effect estimates reporting bias. Quality of evidence will be categorised into "very low', 'low', 'moderate' or 'high'.

\section{ETHICS AND DISSEMINATION}

A formal ethical approval is not required since no primary data of individuals will be collected. The status of the planned review will be updated regularly in PROSPERO. Results from this review will be published in leading peer-reviewed journals in the field of telemedicine and eHealth. Furthermore, results will be presented at international conferences and workshops to facilitate dissemination into clinical practice.

\section{CONCLUSION}

This systematic review and meta-analysis will complement the evidence base of IMIs and allow for an evaluation of their feasibility as aftercare for the tertiary prevention as a significant component of mental healthcare. In case of cavities in research areas or unsatisfactory confirmation, we will suggest future research strategies. The findings will extend previous literature on the effectiveness of IMIs in different areas of healthcare like prevention ${ }^{40}$ 
or as an alternative to face-to-face therapy. ${ }^{55}$ Furthermore, the results will provide clinicians and public health policy makers with a valuable overview of the possibilities of IMIs in monitoring and managing patients after regular treatment and in preventing relapse or readmission.

Correction notice This paper has been amended since it was published Online First. Owing to a scripting error, some of the publisher names in the references were replaced with 'BMJ Publishing Group'. This only affected the full text version, not the PDF. We have since corrected these errors and the correct publishers have been inserted into the references.

Contributors All authors were involved in the concept and review design of the study and data analysis plan; contributed to the further writing and approved the final version of the manuscript. SH and SF wrote the draft of this manuscript. LS provided valuable revisions.

Funding This research received no specific grant from any funding agency in the public, commercial or not-forprofit sectors.

Competing interests None declared.

Provenance and peer review Not commissioned; externally peer reviewed.

Open Access This is an Open Access article distributed in accordance with the Creative Commons Attribution Non Commercial (CC BY-NC 4.0) license, which permits others to distribute, remix, adapt, build upon this work non-commercially, and license their derivative works on different terms, provided the original work is properly cited and the use is non-commercial. See: http://creativecommons.org/ licenses/by-nc/4.0/

(c) Article author(s) (or their employer(s) unless otherwise stated in the text of the article) 2017. All rights reserved. No commercial use is permitted unless otherwise expressly granted.

\section{REFERENCES}

1. Steel Z, Marnane C, Iranpour C, et al. The global prevalence of common mental disorders: a systematic review and meta-analysis 1980-2013. Int J Epidemiol 2014;43:476-93.

2. Paykel ES, Brugha T, Fryers T. Size and burden of depressive disorders in Europe. Eur Neuropsychopharmacol 2005;15:411-23.

3. Yonkers KA, Bruce SE, Dyck IR, et al. Chronicity, relapse, and illness-course of panic disorder, social phobia, and generalized anxiety disorder: findings in men and women from 8 years of follow-up. Depress Anxiety 2003;17:173-9.

4. Olmsted MP, Kaplan AS, Rockert W. Defining remission and relapse in bulimia nervosa. Int J Eat Disord 2005;38:1-6.

5. Pini S, de Queiroz V, Pagnin D, et al. Prevalence and burden of bipolar disorders in European countries. Eur Neuropsychopharmacol 2005;15:425-34.

6. Haywood TW, Kravitz HM, Grossman LS, et al. Predicting the "revolving door" phenomenon among patients with schizophrenic, schizoaffective, and affective disorders. Am J Psychiatry 1995;152:856-61.

7. Mykletun A, Overland S, Dahl AA, et al. A population-based cohort study of the effect of common mental disorders on disability pension awards. Am J Psychiatry 2006;163:1412-8.

8. Simon GE. Social and economic burden of mood disorders. Biol Psychiatry 2003;54:208-15.

9. Joukamaa M, Heliövaara M, Knekt $P$, et al. Mental disorders and cause-specific mortality. Br J Psychiatry 2001;179:498-502.

10. Caplan G. Principles of Preventive Psychiatry. New York: Basic Books, 1964.

11. McFarlane T, Olmsted MP, Trottier K. Timing and prediction of relapse in a transdiagnostic eating disorder sample. Int J Eat Disord 2008;41:587-93.

12. Blank L, Peters J, Pickvance S, et al. A systematic review of the factors which predict return to work for people suffering episodes of poor mental health. J Occup Rehabil 2008;18:27-34.

13. Vittengl JR, Clark LA, Dunn TW, et al. Reducing relapse and recurrence in unipolar depression: a comparative meta-analysis of cognitive-behavioral therapy's effects. J Consult Clin Psychol 2007;75:475-88.

14. Carter JC, McFarlane TL, Bewell C, et al. Maintenance treatment for anorexia nervosa: a comparison of cognitive behavior therapy and treatment as usual. Int J Eat Disord 2009;42:202-7.
15. Beynon S, Soares-Weiser K, Woolacott N, et al. Psychosocial interventions for the prevention of relapse in bipolar disorder: systematic review of controlled trials. $\mathrm{Br} J$ Psychiatry 2008;192:5-11.

16. Scott J, Colom F, Vieta E. A meta-analysis of relapse rates with adjunctive psychological therapies compared to usual psychiatric treatment for bipolar disorders. Int J Neuropsychopharmacol 2007;10:123-9.

17. Geddes JR, Carney SM, Davies C, et al. Relapse prevention with antidepressant drug treatment in depressive disorders: a systematic review. The Lancet 2003;361:653-61.

18. Steffanowski A, Löschmann $\mathrm{C}$, Schmidt J, et al. [Meta-analysis on the effectiveness of psychosomatic rehabilitation. The MESTA-trial]. Huber Bern, 2007

19. MacDonald L, Chapman S, Syrett M, et al. Improving medication adherence in bipolar disorder: A systematic review and metaanalysis of 30 years of intervention trials. J Affect Disord 2016;194:202-21.

20. Barkhof E, Meijer CJ, de Sonneville LM, et al. Interventions to improve adherence to antipsychotic medication in patients with schizophrenia--a review of the past decade. Eur Psychiatry 2012;27:9-18.

21. Ehrenreich MJ, Robinson CT, Glovinsky DB, et al. Medical inpatients adherence to outpatient psychiatric aftercare: a prospective study of patients evaluated by an inpatient consultation liaison psychiatry service. Int J Psychiatry Med 2012;44:1-15.

22. Sibold M, Mittag O, Kulick B, et al. [Predictors of participation in medical rehabilitation follow-up in working patients with chronic back pain]. Rehabilitation 2011;50:363-71.

23. Kobelt A, Nickel L, Grosch EV, et al. [Participation in psychosomatic outpatient care after in-patient rehabilitation]. Psychother Psychosom Med Psychol 2004;54:58-64.

24. Kampman O, Illi A, Poutanen $\mathrm{P}$, et al. Four-year outcome in noncompliant schizophrenia patients treated with or without homebased ambulatory outpatient care. Eur Psychiatry 2003;18:1-5.

25. Lingam R, Scott J. Treatment non-adherence in affective disorders. Acta Psychiatr Scand 2002;105:164-72.

26. Ramana R, Paykel ES, Melzer D, et al. Aftercare of depressed inpatients--service delivery and unmet needs. Soc Psychiatry Psychiatr Epidemiol 2003;38:109-15.

27. Schulz H, Barghaan D, Harfst T, et al. [Mental Healthcare]. Robert Koch-Institut, ed. [Health report of Germany]. Berlin: Robert KochInstitut, 2008

28. Adair CE, McDougall GM, Mitton CR, et al. Continuity of care and health outcomes among persons with severe mental illness. Psychiatr Serv 2005;56:1061-9.

29. World Health Organization (WHO). A health telematics policy.: Report of the WHO Group Consultation on Health Telematics. Geneva, 1998

30. Ebert DD, van Daele T, Nordgreen T, et al. Internet and mobile-based psychological interventions: applications, efficacy and potential for improving mental health in Europe. A report of the EFPA e-health taskforce. Eur Psychol under review.

31. Nordgren LB, Hedman E, Etienne J, et al. Effectiveness and costeffectiveness of individually tailored Internet-delivered cognitive behavior therapy for anxiety disorders in a primary care population: a randomized controlled trial. Behav Res Ther 2014;59:1-11.

32. Hedman E, Andersson E, Ljótsson B, et al. Cost-effectiveness of Internet-based cognitive behavior therapy vs. cognitive behavioral group therapy for social anxiety disorder: results from a randomized controlled trial. Behav Res Ther 2011;49:729-36.

33. Internet Society. Global Internet Report. 2016. http://bit.ly/2fQDYzm (accessed 15 Feb 2017).

34. Barak A, Hen L, Boniel-Nissim M, et al. A Comprehensive Review and a Meta-Analysis of the Effectiveness of Internet-Based Psychotherapeutic Interventions. J Technol Hum Serv 2008;26(24):109-60.

35. Andersson G, Carlbring P, Ljótsson B, et al. Guided InternetBased CBT for Common Mental Disorders. J Contemp Psychother 2013;43:223-33.

36. Richards D, Richardson T. Computer-based psychological treatments for depression: a systematic review and meta-analysis. Clin Psychol Rev 2012;32:329-42.

37. Olthuis JV, Watt MC, Bailey K, et al. Therapist-supported internet cognitive behavioural therapy for anxiety disorders in adults. Cochrane Database Syst Rev 2016;3:290.

38. Kuester A, Niemeyer H, Knaevelsrud C. Internet-based interventions for posttraumatic stress: a meta-analysis of randomized controlled trials. Clin Psychol Rev 2016;43:1-16.

39. Lauder S, Chester A, Castle D, et al. A randomized head to head trial of MoodSwings.net.au: an internet based self-help program for bipolar disorder. J Affect Disord 2015;171:13-21. 
40. Sander L, Rausch L, Baumeister H. Effectiveness of Internet-Based Interventions for the Prevention of Mental Disorders: A Systematic Review and Meta-Analysis. JMIR Ment Health 2016;3:e38.

41. Nieuwenhuijsen $\mathrm{K}$, Faber B, Verbeek JH, et al. Interventions to improve return to work in depressed people. Cochrane Database Syst Rev 2014;12:CD006237.

42. Ebert DD, Hannig W, Tarnowski T, et al. [Web-based rehabilitation aftercare following inpatient psychosomatic treatment]. Rehabilitation 2013;52:164-72.

43. Zwerenz R, Becker J, Gerzymisch K, et al. Evaluation of a transdiagnostic psychodynamic online intervention to support return to work: A randomized controlled trial. PLoS One 2017;12:e0176513.

44. Schmädeke S, Bischoff C. [Effects of Smartphone-supported Rehabilitation Aftercare (eATROS) for depressive patients]. Verhaltenstherapie 2015;25:277-86.

45. Fichter MM, Quadflieg N, Lindner S. Internet-based relapse prevention for anorexia nervosa: nine- month follow-up. J Eat Disord 2013;1:23

46. Bauer S, Wolf M, Haug S, et al. The effectiveness of internet chat groups in relapse prevention after inpatient psychotherapy. Psychother Res 2011;21:219-26.

47. Shamseer L, Moher D, Clarke M, et al. Preferred reporting items for systematic review and meta-analysis protocols (PRISMA-P) 2015: elaboration and explanation. BMJ 2015;349:97647.
48. Kampling $\mathrm{H}$, Baumeister $\mathrm{H}$, Jäckel $\mathrm{WH}$, et al. Prevention of depression in chronically physically ill adults. Cochrane Database Syst Rev 2014.

49. Witkiewitz K, Marlatt GA. Relapse prevention for alcohol and drug problems: that was Zen, this is Tao. Am Psychol 2004;59:224-35.

50. Newman MG, Szkodny LE, Llera SJ, et al. A review of technologyassisted self-help and minimal contact therapies for anxiety and depression: is human contact necessary for therapeutic efficacy? Clin Psychol Rev 2011;31:89-103.

51. Tait RJ, Spijkerman R, Riper H. Internet and computer based interventions for Cannabis use: a meta-analysis. Drug Alcohol Depend 2013;133:295-304.

52. Rooke S, Thorsteinsson E, Karpin A, et al. Computer-delivered interventions for alcohol and tobacco use: a meta-analysis. Addiction 2010;105:1381-90.

53. Higgins JP, Altman DG, Gøtzsche PC, et al. The Cochrane Collaboration's tool for assessing risk of bias in randomised trials. BMJ 2011;343:d5928.

54. Guyatt GH, Oxman AD, Vist GE, et al. GRADE: an emerging consensus on rating quality of evidence and strength of recommendations. BMJ 2008:336:924-6.

55. Andersson G, Cuijpers P, Carlbring P, et al. Guided Internet-based vs. face-to-face cognitive behavior therapy for psychiatric and somatic disorders: a systematic review and meta-analysis. World Psychiatry 2014;13:288-95 